\title{
Utilización de la Inteligencia Artificial en el diagnóstico patológico de edificaciones de valor patrimonial
}

\section{Use of Artificial Intelligence in the pathological diagnosis of historical buildings}

L. A. Chávez-Hernández ${ }^{(*)}$, C. A. Recarey ${ }^{(*)}$, M. M. García-Lorenzo ${ }^{(*)}$, O. López-Jiménez ${ }^{(* *)}$

\section{RESUMEN}

Cada vez más las tecnologías de la información ocupan nuevas áreas del conocimiento y el diagnóstico patológico en las edificaciones no podía ser la excepción, en este trabajo ponemos a disposición de los profesionales de la construcción y específicamente los especializados en este tema un Sistema Experto (SE) que utiliza la Inteligencia Artificial (IA) y un Sistema Basado en el Conocimiento (SBC) para que identifique y diagnostique las patologías que se presentan en los materiales de Piedra Natural y Cerámica utilizados en la construcción. De tal manera que se crea un entorno informatizado para la búsqueda de información, que facilita la actualización o integración de datos relacionados con los deterioros en las edificaciones.

\section{SUMMARY}

More and more often Information Technologies occupy new areas of knowledge, and the pathological diagnosis of buildings is not an exception. In this work, we offer to construction professionals and particularly those who specialize in the topic of pathological diagnosis, an expert system that uses Artificial Intelligence (AI) and a knowledge-based system to identify and diagnose the pathologies in the materials made of natural stone and ceramics used in construction. Thus, a computerized environment is created to search for information that facilitates the updating and integration of data on the extent of damage sustained by buildings.
Palabras clave: Diagnóstico patológico; inteligencia artificial; piedra; cerámica.
Keywords: Pathological diagnosis; artificial system; stone; ceramics.

\footnotetext{
(*) Universidad Central "Marta Abreu" de Las Villas (Cuba).

(*) Universidad "José Martí", Sancti Spíritus (Cuba).

Persona de contacto/Corresponding author: chavez@uclv.edu.cu. (J. A. Chávez-Hernández).
}

Recibido/Received: 05 apr 2011 Aceptado/Accepted: 01 mar 2012 Publicado online/ Published online: 12 jul 2012 


\section{INTRODUCCIÓN}

La importancia de la preservación de nuestro patrimonio en este caso, el arquitectónico, surge de su valor como testimonio de distintos fenómenos culturales, y su acción como elemento que mantiene la cohesión de un grupo. Manifiesta también, los valores desarrollados en el tiempo como acciones válidas de un proceso histórico, y que aún pueden serlo en el futuro.

Hasta el momento los arquitectos e ingenieros de la construcción han alcanzado gran desarrollo; y para apoyar a estos profesionales múltiples herramientas informáticas se han desarrollado, sin embargo, se desconoce de la existencia de trabajos previos que apoyen la toma de decisiones en el diagnóstico patológico en las edificaciones, y por ende se está desaprovechando el potencial de la informática para hacer trabajos de diagnóstico de forma más rápida y eficiente, evitando perder la experticia acumulada.

El problema que nos ocupa entonces es desarrollar un software que permita el análisis y diagnóstico patológico en edificaciones -en este caso tomamos la Piedra ${ }^{1}$ y la Cerámica ${ }^{2}$ como punto de partida-, utilizando como herramienta de análisis la Inteligencia Artificial.

\section{DESARROLLO}

En el año 1956, durante un Congreso en Dartmouth (U.S.A.) se propuso el término de Inteligencia Artificial para agrupar a todos los métodos, técnicas e intentos de simular el intelecto humano en la computadora. Casi todos los especialistas están de acuerdo con esto, y quien acuñó este término fue el matemático John McCarthy ${ }^{3}$. Además de McCarthy, son considerados padres de esta disciplina Marvin Minsky ${ }^{4}$ y Herbert Simon y Allen Newell de la Universidad de Carnegie-Mellon.

1 Ámbito de los materiales de la construcción, se aplica a las rocas presentes en la corteza terrestre, después de su extracción y elaboración por la mano del hombre; en consecuencia se encuentra incluido dentro del término "material rocoso" o "roca".

${ }^{2}$ Se deriva del vocablo griego keramos, cuya raíz sánscrita significa "quemar". En su sentido estricto se refiere a la arcilla en todas sus formas. Sin embargo, el uso moderno de este término incluye a todos los materiales inorgánicos no metálicos que se forman por acción del calor.

${ }^{3}$ Creador del LISP, profesor del Instituto de Tecnología de Massachusetts (MIT).

${ }^{4}$ Profesor del Instituto de Tecnología de Massachusetts (MIT)
En la década del 70 se reconoció que los métodos de solución de problemas generales eran insuficientes para resolver los problemas orientados a aplicaciones -como por ejemplo la Inteligencia Artificial por sí sola-, se determinó que era necesario el conocimiento específico sobre el problema, limitado a los dominios de aplicación de interés, en lugar de conocimiento general aplicable a muchos dominios. Este reconocimiento condujo al desarrollo de Sistemas Basados en el Conocimiento (SBC), donde un experto es capaz de utilizar este conocimiento básico para reconocer rápidamente rasgos sobresalientes del problema, de ahí que la potencia de un SBC radica precisamente en el conocimiento que posee.
En términos generales, un SBC puede ser definido como:

Un sistema computarizado que usa conocimiento sobre un dominio para arribar a una solución de un problema de ese dominio. Esta solución es esencialmente la misma que la obtenida por una persona experimentada en el dominio del problema cuando se enfrenta al mismo problema (1).

Según Zenaida García (2), un SBC se puede definir como: "Un sistema informático que simula el proceso de aprendizaje, de memorización, de razonamiento, de comunicación y de acción de un experto humano en una determinada rama de la ciencia, suministrando, de esta forma, un consultor que puede sustituirle con unas ciertas garantías de éxito".

Los SBC son de gran importancia hoy en día, debido al aumento en los volúmenes de información y el grado de completitud y complejidad de la misma.

Entre algunos campos donde con más frecuencia se aplican se encuentran (3):

- Medicina: Donde su función es realizar diagnósticos de enfermedades basados en el cálculo de probabilidades. (3). MYCIN. Diagnóstico y terapia de enfermedades infecciosas bacterianas, creado en la Universidad de Stanford. Varios test han demostrado que MYCIN trabaja tan bien o mejor que un médico.(1). EMYCIN. Es una versión independiente del dominio de MYCIN, que contiene todo lo de este último, excepto su conocimiento sobre enfermedades infecciosas de la sangre, facilitando el desarrollo de aplicaciones de diagnósticos de otros tipos. CASEY (4), para diagnosticar problemas cardiacos. SHRINK (5), para el diagnóstico en psiquiatría. PROTOS (6), para el diagnóstico de trastornos auditivos.

- Industria: El reto de los Sistemas Expertos industriales se centra en la necesidad de que se comuniquen con dispositivos sensores, bases de datos, dispositivos de mando y accionamiento en tiempo real. CSA Manejo de centrales nucleares. KRITIK: (7) para el diseño de pequeños ensambles mecánicos. XBE: (8), para diseñar sistemas de manufactura.

- Electrónica: Se orientan al diseño, diagnóstico y reparación. El uso de Sistemas Expertos se debe a la creciente complejidad de los circuitos y al gran número de parámetros a considerar en los mismos. DAA: Diseño de circuitos con alto grado de integración. ARGOS-2: Simulación de la toma de decisión de un robot. 
- Aeronáutica: Orientado al control de la posición de los satélites y la interpretación de sus imágenes. AIRPLAN: Planificación de lanzamientos y despegues, recuperación de aviones sobre una base o un portaaviones. CAT: Evaluación de una amenaza de abordo de un avión.

- Agricultura: Diagnóstico y tratamiento de tierras, control de plagas, creación de nuevos herbicidas y tratamiento de animales. VP-EXPERT:(9), diagnóstico de plagas insectiles en el maíz. TOMATEX ver.3: aplicado en el tomate averigua los desórdenes más típicos en las plantas para poder dar unas especificaciones para su solución.

- Química: Estudio de las propiedades de compuestos, interpretación de los resultados obtenidos en los análisis, planificación de los procesos de síntesis. DENDRAL: Es un sistema que determina la estructura molecular de los compuestos químicos a partir de una información primaria, procedente de ensayos.

El panorama de experiencias de inteligencia artificial y de sistemas expertos en arquitectura es bastante desolador, mientras que en otros campos la aplicación de técnicas de I.A. está extendida en arquitectura existen muy pocas experiencias. En un artículo de reflexión sobre las posibilidades de la I.A. en Ingeniería y Arquitectura, Garret (10) aboga por la creación de aplicaciones de este tipo y consideran que la falta de iniciativa es debida a varias razones, entre las que destacan: el carácter conservador que en cuestiones de seguridad tiene la disciplina, la falta de representaciones del conocimiento sobre la concepción y diseño de estructuras reales, la necesidad de equipos multidisciplinares para solventar la complejidad que subyace en toda estructura. Por ello, ha sido más fácil desarrollar software que se limite al análisis del problema que elaborar sistemas expertos de apoyo.

Ejemplos de sistemas expertos para el apoyo a los ingenieros son:

- Sistemas de ayuda en la definición del modelo de análisis estructural: Una de las primeras experiencias fue el sistema SACON (11) que pretendía aconsejar sobre la estrategia de análisis para estructuras de barras. En la misma línea pero para elementos finitos se encuentran dos experiencias, FEAA-Dolsak (12) y Turkiyyah y Fenves (13) También se incluiría a Hartmann y Lehner (14) describiendo un sistema para ayudar en la selección de la estrategia numérica a seguir en problemas de optimización.
- Sistemas de ayuda en el diseño basados en experiencia heurística: Por ejemplo Mukherjee and Deshpande (15) definen una red neuronal en lugar de un sistema experto para ayudar en el diseño. El artículo cita en contra de los sistemas expertos: la falta de aprendizaje, las reglas deben ser claras y el necesario conocimiento profundo de ingeniería. Por ello aboga por una red neuronal con reglas de conocimiento. En otro artículo Yoshimura (16) definen un sistema experto para el diseño de componentes nucleares y comparan el diseño obtenido con conocimientos heurísticos y el obtenido con una metodología clásica de optimización. En cuanto al razonamiento cualitativo puede citarse Bozzo (17) con trabajos en el diseño sismo resistente de edificios.

- Otro ejemplo lo es un sistema experto para programar la ejecución de grandes proyectos de vivienda de Álvarez-Romero (18). Este trabajo presenta una propuesta de un sistema informatizado para facilitar este proceso de la programación de la ejecución de un proyecto de vivienda masiva.

\subsection{Planteamiento del problema}

Se requiere entonces llevar a cabo una identificación y diagnóstico de diferentes patologías (19) en los materiales de Piedra Natural y Cerámica utilizados en la construcción. Dichas patologías pueden presentarse en cualquier elemento constructivo ${ }^{5}$ de las edificaciones y cada una con diferentes Síntomas, o Condiciones Propicias del lugar, pero para llevar este diagnóstico es necesario antes conocer las diferentes formas de deterioro en los materiales pétreos y cerámicos (20), es decir, adquirir el conocimiento.

\subsection{Proceso de adquisición del conoci- miento para el diagnóstico de patolo- gías en edificaciones}

Para la adquisición del conocimiento nos basamos en el propio proceso patológico (21) donde este establece que para atacar una enfermedad, el médico requiere, en primer lugar, su diagnóstico. También en la ingeniería, para atacar un problema constructivo, es necesario "diagnosticarlo", es decir, conocer su proceso, su origen, sus causas, su evolución, sus síntomas y su estado actual, y adquirir los datos de forma inversa, es decir, según el estudio patológico (Figura 1).

Según este proceso nos dimos a la tarea de crear una Base de Datos en el programa Excel de Windows que aglutinara todas las patologías que se presentan en las edificaciones
${ }^{5}$ Un elemento estructural lo son los componentes de las edificaciones como: pisos, muros, arcos, bóvedas, columnas, etc. 
1. Fases del Proceso Patológico.

2. Esquema la estructura de la Base de Conocimiento.

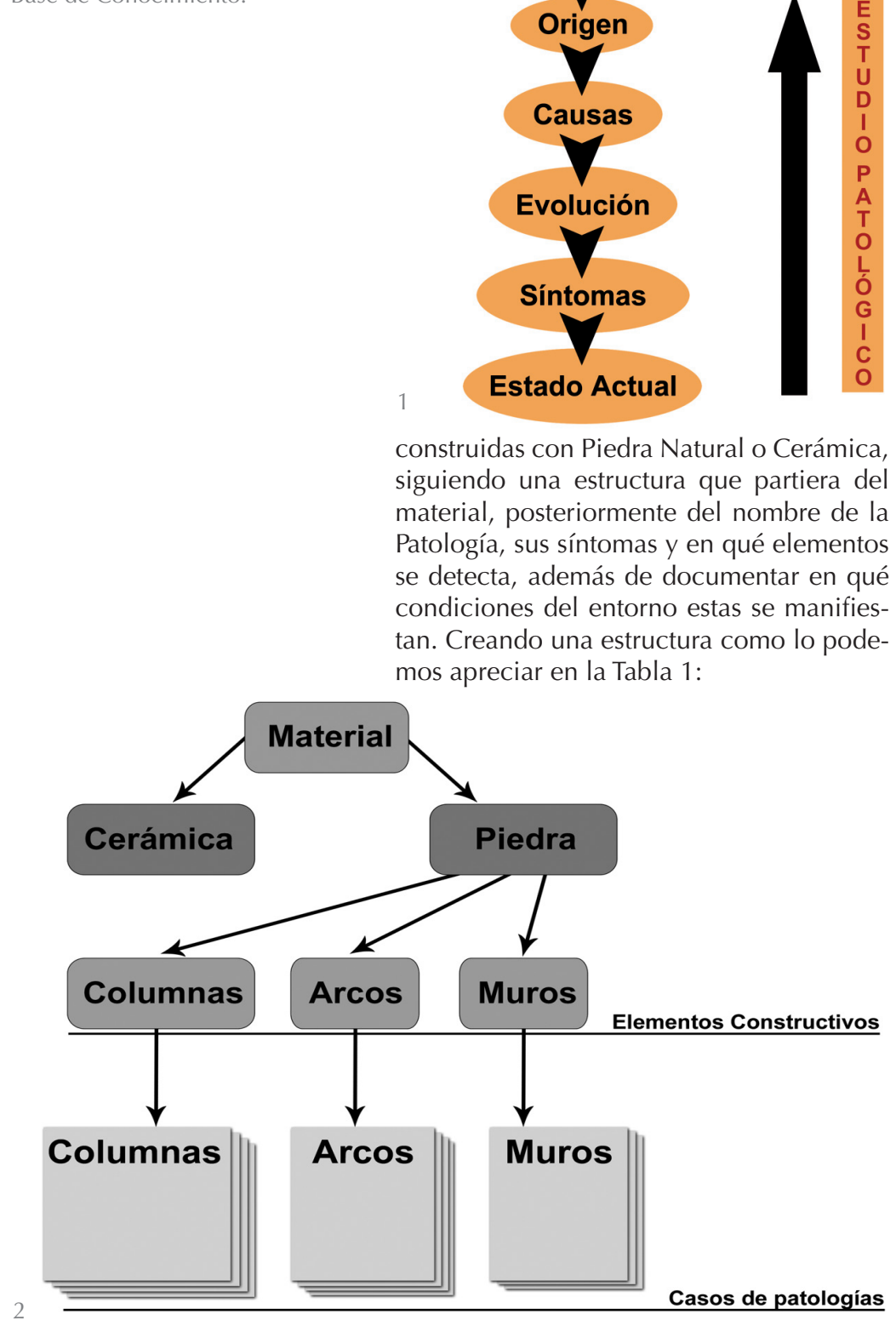

En esta Base de Datos además se incluyeron imágenes identificadoras de cada una de las patologías, descripciones de las lesiones y mayor información sobre sus posibles causas, así mismo cómo deben instrumentarse estas para obtener datos de su comportamiento y forma de modelarse al introducirlas en un modelo matemático para analizar su acción en la estructura. En el caso de la Cerámica pudimos registrar 10 patologías que se presentan en los diferentes elementos constructivos y para la Piedra registramos 9, basándonos para este registro en las coincidencias sobre el tema de las siguientes fuentes consultadas: (20-42) además de la experiencia obtenida en soluciones a edificaciones del casco histórico de las ciudades de La Habana, Santa Clara y Cienfuegos en nuestro país, Cuba.

\subsection{Construcción de la Base de Conoci- miento y fichero para almacenarla}

Una vez obtenida la Base de Datos con las patologías que se manifiestan en los materiales estudiados se hizo necesario "traducir" esta Base de Datos a una Base de Conocimiento, empleando un fichero que sea "comprendido" por los métodos de I.A. a aplicar.

La Base de Conocimiento fue concebida y estructurada jerárquicamente como un árbol (Figura 2), donde en el nivel raíz se encuentra el material y atendiendo a este el elemento constructivo, y en las hojas del árbol las patologías que se manifiestan en los mismos.

Esta forma de estructurar el Conocimiento nos permitió crear Casos en un fichero que organizamos en tres secciones:

1. Sección de completitud de la base de casos: Es una bandera que puede ser 0 "no la presenta" o 1 "la presenta".

Tabla 1. Fragmento de la forma en que se organizó el Conocimiento sobre Patologías que se presentan en el material Cerámica

\begin{tabular}{|c|c|c|c|}
\hline \multicolumn{4}{|c|}{ Material: Cerámica } \\
\hline Patologías & Síntomas & $\begin{array}{l}\text { Elementos } \\
\text { Constructivos }\end{array}$ & Condiciones del Entorno \\
\hline Eflorescencias & $\begin{array}{l}\text { Manchas blancas } \\
\text { Manchas amarillas } \\
\text { Manchas marrones } \\
\text { Manchas rojizas } \\
\text { Manchas verdes } \\
\text { Decoloración }\end{array}$ & $\begin{array}{l}\text { Muros } \\
\text { Cubierta } \\
\text { Pisos } \\
\text { Enchapes } \\
\text { Rasillas }\end{array}$ & $\begin{array}{l}\text {-Está ubicada en lugar húmedo. } \\
\text {-Posee Manchas hasta 1,2 m de altura. } \\
\text {-Existen marcados cambios de temperaturas } \\
\text { en el transcurso del día. } \\
\text {-Exposición a la lluvia y al hielo. } \\
\text { - Ubicación en dirección al sentido del viento. }\end{array}$ \\
\hline Erosión & $\begin{array}{l}\text { Degradación } \\
\text { Destrucción } \\
\text { Desprendimiento } \\
\text { Pérdida de volumen }\end{array}$ & $\begin{array}{l}\text { Muros } \\
\text { Cubierta } \\
\text { Pisos } \\
\text { Muretes } \\
\text { Rasillas } \\
\text { Jardineras }\end{array}$ & $\begin{array}{l}\text {-Existen marcados cambios de temperaturas en el transcurso del día. } \\
\text {-Existen temperaturas menores de } 0^{\circ} \mathrm{C} \text {. } \\
\text {-Exposición a los agentes meteorológicos. } \\
\text {-Presenta zonas de escorrentías } \\
\text { - Ubicación en dirección al sentido del viento. } \\
\text {-Presenta irregularidades en la superficie del elemento. } \\
\text { - Ha sido víctima de algún siniestro. } \\
\text {-Existe la presencia de hongos. }\end{array}$ \\
\hline
\end{tabular}


2. Sección de las patologías que posee base de casos: Es un arreglo ordenado según la ubicación de las patologías en la base de casos, y donde se le asigna un número (id) a las patologías en sus diferentes manifestaciones.

La base de casos: Es una matriz binaria hasta la columna n-1 y en la columna n posee el id de la patología. Dicha matriz se organiza de la siguiente manera: una lista de síntomas $\left(\mathrm{S}_{1}, \mathrm{~S}_{2}, \mathrm{~S}_{3}, \ldots \mathrm{S}_{k}\right)$. Una lista de condiciones propicias $\left(\mathrm{C}_{1}, \mathrm{C}_{2}, \mathrm{C}_{3}, \ldots \mathrm{C}_{j}\right)$; donde, $k$ es la cantidad máxima de síntomas (La sumatoria de todos los síntomas en todas las patologías) y j la cantidad máxima de condiciones propicias (La sumatoria de todas las condiciones propicias en todas las patologías). El esquema de la matriz para confeccionar los casos es demasiado grande como para mostrarlo en este documento, en la siguiente Tabla 2 brindamos un fragmento.

Tabla 2.

Esquema de la matriz en la Base de Casos

\begin{tabular}{|c|c|c|}
\hline$S_{1} S_{2} S_{3} \ldots . . S_{K}$ & $\mathrm{C}_{1} \mathrm{C}_{2} \ldots \ldots \mathrm{C}_{\mathrm{J}}$ & PATOLOGÍA \\
\hline $\begin{array}{lllllll}1 & 0 & 1 & \ldots & & 0\end{array}$ & $\begin{array}{llll}1 & 1 & \ldots & 0\end{array}$ & 3 \\
\hline $\begin{array}{lllll}0 & 0 & 1 & \ldots & 1\end{array}$ & $\begin{array}{llll}1 & 1 & \ldots & 1\end{array}$ & 4 \\
\hline $\begin{array}{llllll}1 & 0 & 0 & \ldots & 0 \\
\end{array}$ & $\begin{array}{llll}0 & 1 & \ldots & 1\end{array}$ & 0 \\
\hline . . . & . . & . \\
\hline . . & . & . \\
\hline $\begin{array}{lllll}1 & 1 & 1 & \ldots & 1\end{array}$ & $\begin{array}{llll}1 & 1 & \ldots . & 0\end{array}$ & 12 \\
\hline
\end{tabular}

Para ilustrar mejor esto ponemos un ejemplo a continuación:

La patología Costras y Depósitos en el material Cerámica, en el elemento constructivo Muros, se pueden manifestar por uno o varios de los siguientes Síntomas: Costras Negras, Ensuciamiento, Acumulación de Partículas, Caparazón, Incrustaciones. Es decir, esta patología puede manifestarse por hasta 5 Síntomas y cumpliéndose además al menos una o varias de las siguientes Condiciones Propicias: Existe la presencia de calefacciones, Se encuentra en una zona de tráfico vehicular, Se encuentra en una zona de procesos industriales, Es un lugar húmedo, Presenta zonas de escorrentías, Está ubicada en lugares con presencia continua de agua. Es decir, pueden existir hasta al menos 6 Condiciones que pueden propiciar que esta patología se manifieste.

Este caso entonces de presencia de Costras y Depósitos en Muros de Cerámica en la matriz quedaría de la siguiente forma: Los síntomas Costras Negras, Ensuciamiento, Acumulación de Partículas, Caparazón e Incrustaciones se marcarían con el valor 1 porque la "presenta", así mismo pasa para las Condiciones Propicias ya mencionadas en el párrafo anterior, se marcarían igual con el valor 1 , se marcarían con 0 todos los Síntomas o Condiciones Propicias que este caso "no presenta".

Hasta el momento de terminar esta investigación el número de Síntomas de las patologías en el material Piedra eran de 82 y en la Cerámica de 37, las Condiciones Propicias para Piedra eran de 62 y para Cerámica de 32, es decir, que la matriz de la Base de Casos de la Cerámica presenta 70 columnas y la de Piedra 145 columnas, incluyendo el Id que identifica la patología. No obstante podremos adicionar más Síntomas o Condiciones cuando deseemos como lo podremos apreciar más adelante.

\subsection{Editor de Base de Conocimientos y sus facilidades}

Se implementó un editor (software) de Base de Conocimientos para la confección de los casos puesto que disminuye el tiempo de ingresar conocimiento y la posibilidad de errores debido a la gran cantidad de Casos.

Con la ayuda del editor (Figura 3) el experto puede generar rápidamente los casos, editar los Rasgos Generales a combinar y la Información Complementaria ${ }^{6}$ de cada patología con facilidad. Realizar operaciones de inserción y eliminación de casos de una forma eficiente. Listar los casos existentes en la base. Modificar el contenido de rasgos predictores existentes. Obtener salvas como actualizaciones del sistema completo, referenciando las BC en uso o salvadas para evitar la pérdida de información teniendo la posibilidad de trabajar con diferentes $\mathrm{BC}$ en dependencia de lo que desee el experto.

El Editor de BC, está dividido en cinco secciones como se muestra en la Figura 3.

1. Sección de entrada de datos (campo 1), en esta sección se introduce según el Material y el Elemento Constructivo, los Síntomas y las Condiciones Propicias la Patología que reúne estas características.

2. Sección de mostrar los resultados (campo 2), cuando se accede a la misma se muestra una ventana como se muestra en la Figura 4. En esta sección se muestran dos tablas: la primera con la base de casos que se va llenando actualmente (campo 1) y otra con la BC temporal del sistema (campo 2) si este posee alguna para el elemento estructural correspondiente. Posee además tres botones: dos para eliminar instancias de ambas tablas (campos 3 y 4) respectivamente, y un tercero para agregarle instancias de la BC temporal para la BC del sistema (campo 5).
${ }^{6}$ Son aquellos que podemos utilizar para una mejor comprensión de la Patología estudiada como su Descripción, las Causas que la provocan, los Ensayos que podemos realizarles para obtener datos y conocer su magnitud, así como diferentes ejemplos de Imágenes con las cuales podemos comparar nuestro Caso y asegurarnos que el resultado de la búsqueda con los valores entrados al sistema son los correctos. 

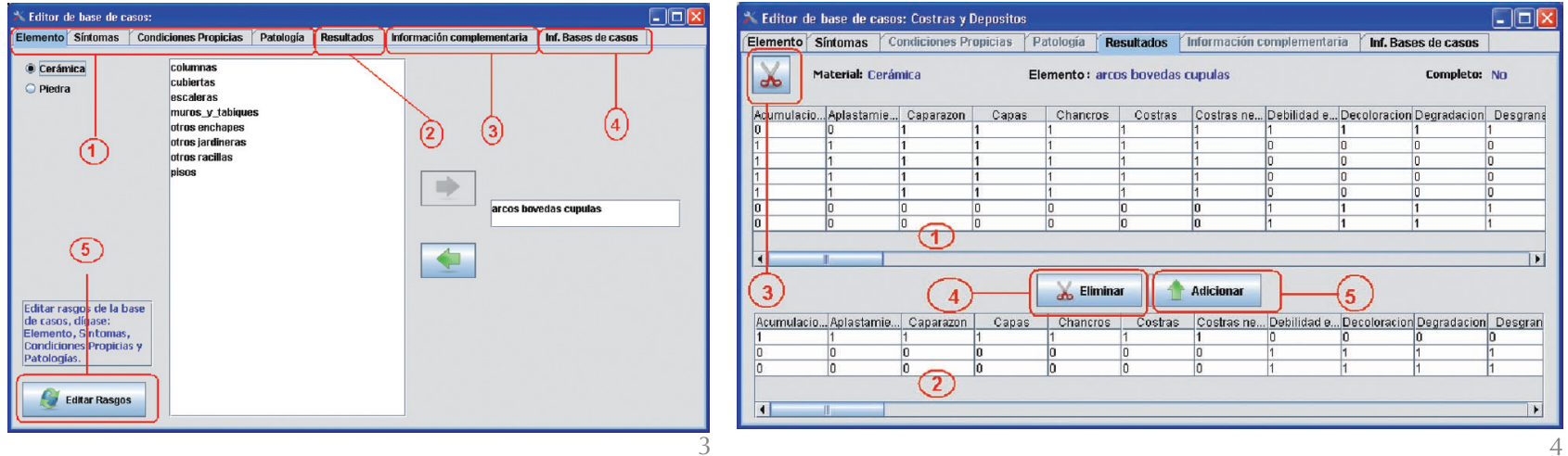

Editor de la Base de Casos.

4. Editor de la Base de Casos: Resultados.

${ }^{7}$ Salvas o copias de seguridad.

${ }^{8}$ La distancia de Hamming se denomina así gracias a su inventor Richard Hamming, profesor de la Universidad de Nebraska
En esta sección se muestran información del material que se está utilizando, el entorno de trabajo e información de la completitud de la BC.

3. Sección para la entrada de los Rasgos o Información Complementaria (Campo 3. Figura 3) de las Patologías como Descripción, las Causas que la provocan, los Ensayos que podemos realizarles para obtener datos y conocer su magnitud, así como podemos entrarle diferentes imágenes de casos que ya se han estudiado en trabajos previos.

4. Sección para la Información de la Base de Casos (Campo 4. Figura 3), en esta el usuario puede ver una lista de backups ${ }^{7}$ de la BC, donde puede ponerles Nombres, la fecha de creación, así como ver el tamaño en MB y cuál es la que está usando.

5. Sección para la Edición de los Rasgos (Campo 5. Figura 3), en esta sección aparece otra ventana donde el usuario puede modificar, agregar o quitar los Rasgos con los cuales se realizan los Casos de cada patología según el material, estos Rasgos son las listas de los Elementos Constructivos, los Síntomas y las Condiciones Propicias.

\subsection{Solución del problema}

Para determinar el método de solución a emplear partimos precisamente de cómo resuelve el experto este problema. Para lo cual el experto busca el nivel de similitud que tiene la nueva edificación a diagnosticar con los casos anteriormente concebidos.

Se trata entonces de hallar el caso más parecido en la Base de Conocimientos al que traemos previamente del trabajo en la edificación.

Al existir en la Base de Casos un caso de cada patología se decidió implementar el Vecino más Cercano. Una característica importante e interesante del Vecino más Cercano es que el método puede cambiar radicalmente sus resultados de clasificación sin modificar su estructura, solamente cambiando la métrica utilizada en el cálculo de la distancia. Por lo tanto, los resultados pueden variar tantas veces como métodos de hallar distancia entre instancias haya. La métrica debe seleccionarse de acuerdo al problema que se desee solucionar.

\subsubsection{Función de distancia}

La distancia es el criterio de comparación principal usado en los métodos basados en vecindad.

$$
\begin{gathered}
d H(A, B)=\sum_{\mathrm{i}} d\left(a_{\mathrm{i}}, b_{\mathrm{i}}\right) \\
d\left(\mathrm{a}_{\mathrm{i}}, b_{\mathrm{i}}\right)=\left\{\begin{array}{l}
1 \text { si } a_{\mathrm{i}}=b_{\mathrm{i}} \\
0 \text { si } a_{\mathrm{i}} \neq b_{\mathrm{i}}
\end{array}\right.
\end{gathered}
$$

La distancia de Hamming ${ }^{8}(\mathrm{dH})$ está descrita en las ecuaciones [1] y [2], donde se tiene en cuenta que se comparan dos objetos $A=\left(a_{1}, a_{2}, \ldots, a_{n}\right)$ y $B=\left(b_{1}, b_{2}, \ldots, b_{n}\right)$, de igual tamaño, donde se comparan cada uno de sus elementos. Esta fue la distancia seleccionada para el desarrollo del SE, por cumplir con los requerimientos para el problema. Ejemplo: La distancia de Hamming entre 1011101 y 1001001 es 2. Obsérvese entonces que mientras mayor es la distancia, menos similitud tienen los casos.

\subsubsection{Inferencia de la solución al caso planteado}

Para buscar el caso más cercano en la BC al que Ud. está entrando se siguen los siguientes pasos:

1. Se selecciona un material, esto posibilita la carga de la lista de rasgos referente al material.

2. Se selecciona un elemento estructural o entorno aquí es donde se carga la BC correspondiente al mismo.

3. Se editan los valores de los rasgos predictores correspondientes a la edifica- 
ción a diagnosticar (lista de síntomas y lista de condiciones propicias). Esto produce que se cree una nueva instancia pero sin asociarle una clase o patología.

4. Se calcula la distancia de Hamming de todos los valores de la instancia a clasificar con toda la BC.

5. Se selecciona el mínimo de las distancias y se le asocia una clase a la nueva instancia que se corresponde con la clase de los casos donde se alcanzó distancia mínima y se devuelve el nivel de similitud asociado a la misma.

\subsubsection{Explicación de la solución}

En el caso del nivel de similitud en cualquier momento podemos decirle cuáles patologías mostrar, es decir, este valor lo podemos cambiar, según nuestras simulaciones por encima del $85 \%$ de similitud los resultados son satisfactorios, dando como respuesta la patología que se trataba identificar y en algunos casos 2 pues las diferencias eran mínimas. Por debajo del $85 \%$ ya se mostraban respuestas de hasta 3 o más patologías.

Ilustremos la solución con un ejemplo: En una edificación nos encontramos que en una zona de un Muro (Elemento) (Figura 5) de Piedra (Material) se aprecian manchas amarillentas, además de decoloración, manchas negras y manchas blancas tenemos entonces 4 síntomas identificadas en esa zona. Así mismo podemos apreciar que esa zona se encuentra expuesta al agua de lluvia, que la obra posee mucho tiempo de uso, y que además por los alrededores hay abundante tráfico vehicular, tenemos entonces 3 condiciones que pueden propiciar la patología o patologías que queremos identificar.

En la lista de Síntomas de las patologías para la piedra estarían marcados con 1 sólo 4 de ellos, los demás serían marcados con 0 , así mismo en la lista de Condiciones Propicias estarían marcados con 1 sólo 3 y los demás con 0 . Es decir que contaría con 7 rasgos marcados con 1 de las 144 posibles, veamos cómo se halla el Vecino más Cercano en la Base de Conocimiento:
Dónde:

Síntomas: $S_{1}-$ Hendiduras, $S_{2}-$ Manchas Amarillentas, $\mathrm{S}_{3}$ - Manchas Blancas, $\mathrm{S}_{4}-$ Decoloración, $\mathrm{S}_{5}$ - Láminas, $\mathrm{S}_{\mathrm{k}}$ - Manchas Negras

Condiciones Propicias: $\mathrm{C}_{1}$ - Presencia de Cemento Carbonatado, $\mathrm{C}_{2}$ - Agua de Lluvia, $\mathrm{C}_{3}$ - La Obra posee mucho tiempo de uso, $\mathrm{C}_{4}$ - Presencia de Escamas, $\mathrm{C}_{5}$ - Abundante Tráfico Vehicular, $\mathrm{C}_{\mathrm{j}}$ - Presenta Irregularidades en la Superficie del elemento.

Patología: $\mathrm{Id}_{1}$ - Depósito Superficial, $\mathrm{Id}_{2}$ - Pátinas, $I_{d_{3}}$ - Costras, $I_{\mathrm{n}}$ - Eflorescencias

Caso de Entrada:

01110...101101...0

Casos de la BC:

$10001 \ldots 000101 \ldots 1$ Depósito Superficial $\left(\operatorname{ld}_{1}\right)$

01010...101101...0 Pátinas $\left(\mathrm{Id}_{2}\right)$

$00001 \ldots 000101 \ldots 0$ Costras $\left(\mathrm{Id}_{3}\right)$

00100...000000...0 Eflorescencias $\left(\operatorname{Id}_{\mathrm{n}}\right)$

La mayor distancia posible para este ejemplo con sólo esta pequeña cantidad de Síntomas y Condiciones a tener en cuenta, sería de 12, y recordemos que a mayor distancia menor es la coincidencia de los casos. Así entonces la distancia entre el Caso de Entrada y el 1 (Depósito Superficial) sería de $8(33,34 \%)$. Para el 2 (Pátinas) sería de 1 (91,67\%). Para el 3 (Costras) sería de 6 (50\%). Para el $n$ (Eflorescencias) sería de 6 (50\%). Como podemos apreciar el Caso de Entrada tiene una similitud del $91,67 \%$ con el Caso 2 ya que la distancia entre ellos es de 1, es decir, con la Patología Pátinas, en este caso el programa no sólo devuelve que es la Pátina la patología que se presenta en su caso por estar por encima del $85 \%$ de similitud sino también le dice qué tipo de Pátina puede ser porque las manchas amarillentas son sólo específicas de las Pátinas Bióticas y las manchas negras de las Pátinas negras, esto es muy importante ya que ambas se tratan de forma diferente a la hora de trabajar en su solución. También podemos apreciar que con mucho menos importancia existen presencias de Costras y Eflorescencias.

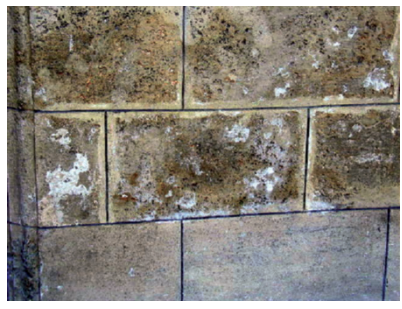

5. Porción de un muro exterior del Edificio: Palacio de los Capitanes Generales. La Habana. Cuba.

Tabla 3. Ejemplo de Matriz para el caso que estamos analizando

\begin{tabular}{|c|c|c|c|c|c|c|c|c|c|c|c|c|c|c|}
\hline $\mathbf{S}_{1}$ & $\mathbf{S}_{\mathbf{2}}$ & $\mathbf{S}_{3}$ & $\mathbf{S}_{\mathbf{4}}$ & $\mathbf{S}_{\mathbf{5}}$ & $\cdots$ & $\mathbf{S}_{\mathrm{K}}$ & $\mathbf{C}_{\mathbf{1}}$ & $\mathbf{C}_{2}$ & $\mathbf{C}_{3}$ & $\mathbf{C}_{\mathbf{4}}$ & $\mathbf{C}_{5}$ & $\cdots$ & $\mathbf{C}_{\mathbf{J}}$ & $\mathbf{I D}$ \\
\hline 1 & 0 & 0 & 0 & 1 & $\cdots$ & 0 & 0 & 0 & 1 & 0 & 1 & $\cdots$ & 1 & 1 \\
\hline 0 & 1 & 0 & 1 & 0 & $\cdots$ & 1 & 0 & 1 & 1 & 0 & 1 & $\cdots$ & 0 & 2 \\
\hline 0 & 0 & 0 & 0 & 1 & $\cdots$ & 0 & 0 & 0 & 1 & 0 & 1 & $\cdots$ & 0 & 3 \\
\hline 0 & 0 & 1 & 0 & 0 & $\cdots$ & 0 & 0 & 0 & 0 & 0 & 0 & $\cdots$ & 0 & $\mathrm{~N}$ \\
\hline
\end{tabular}




\subsection{Recuperación de nuevos casos hacia la BC}

Un razonador basado en casos es bueno sólo en la medida de los casos que este pueda recordar. La recuperación de nuevos casos hacia la BC permite la retroalimentación del sistema y la actualización del mismo, en la misma manera en que el usuario experto se enfrente a problemas reales o simulados. Esta tarea se realiza de manera independiente a la de actualizar la $\mathrm{BC}$ desde el editor de conocimientos puesto que esta tarea se realiza de forma automática, primero si cumple con un nivel de similitud por encima del $85 \%$ y segundo si el usuario está de acuerdo con la solución que arrojó el sistema. Para estos casos se conforma un fichero temporal, los cuales serán adicionados a la base de casos si el experto da su aceptación.

\subsection{Aplicación de la herramienta para su validación}

La Herramienta para su validación se aplicó en edificaciones ya estudiadas anteriormente por el equipo de trabajo de la Facultad de Construcciones de la Universidad Central "Marta Abreu" de Las Villas del cual los autores de esta investigación el Msc. Arq. José A. Chávez Hdez y el Dr. Ing. Carlos A. Recarey Morfa son miembros. Contábamos con todo el levantamiento patológico y fotográfico de varias edificaciones de importancia nacional, como son el Palacio de los Capitanes Generales en La Habana Vieja, las Escuelas de Arte de Cubanacán en ciudad de La Habana, y el Yacht Club de la ciudad de Cienfuegos, estos dos últimos obtuvieron
Premios Nacionales de Restauración. Entramos los datos obtenidos del levantamiento en la Herramienta para comparar sus resultados con los que ya teníamos del estudio que llevamos a cabo, en todos los casos el software coincidió con los resultados que ya habíamos obtenido.

Actualmente lo estamos aplicando en nuevas edificaciones que se encuentran en fase de estudio.

\section{CONCLUSIONES}

Si bien la problemática de las lesiones en la construcción es tan vieja como la construcción misma, el estudio de dicha problemática, es decir la "Patología de las Construcciones" no logra cobrar la difusión necesaria para prevenir la existencia de una Base de Conocimientos que abarque todo el contenido. Con este trabajo se logra desarrollar un Sistema Experto de ayuda al diagnóstico patológico en las edificaciones. Tras el proceso de Ingeniería del Conocimiento se construye una Base de Conocimiento jerárquicamente estructurada por Elemento Constructivo y Material, donde cada caso se describe en función de rasgos principales y complementarios. Se implementa el algoritmo del vecino más cercano con distancia Hamming. La implementación ofrece facilidades de modificación tanto de la función de distancia. Se logró que el diseño e implementación de la interfaz de usuario fuese sencilla, agradable y amigable, para permitir la rápida familiarización con el uso de la herramienta y los procesos de funcionamiento una vez que se encuentre instalada.

\section{BIBLIOGRAFÍA}

(1) Bello, R.: Aplicaciones de la Inteligencia Artificial, Universidad de Guadalajara, Guadalajara, 2002.

(2) García, Z.; Bonet, I. y Cabrera, N. E.: "Sistema de diagnóstico para la estimación de secciones en fallo en sistemas eléctricos de potencia", 2006.

(3) Peña Ayala, A.: Sistemas basados en Conocimiento: Una Base para su Concepción y Desarrollo, Mexico, 2006, p. 199.

(4) Koton, P.: Using experience in learning and problem solving, Computer Science Dept (MIT), 1988.

(5) Kolodner, J. L. y Kolodner, R.: Using experience in clinical problem solving: Introduction and framework, vol. 3, p.11, 1987.

(6) Bradtke, S. y W. G. Lehnert: "Some experiments with case-based search", 1988.

(7) Goel, A.: Integration of case-based reasoning and model-based reasoning for adaptive design problem solving, The Ohio State University, 1989.

(8) Pankakoski, J. et al.: "Applying case-based reasoning to manufacturing systems design", vol. 4, n 4, 1991.

(9) Merino Cisneros, F. L.: "Sistema Experto para Diagnóstico de Plagas Insectiles de Maíz", Agronomía Mesoamericana, vol. 2 (1991).

(10) Garret: "Sistemas CAD en microcomputadoras. Problemática y posibilidades" (1996), p. 4.

(11) Bennett, J. et al:: "A Knowledge-based consultant for structural analysis", Computer Science Department. School of Humanities and Sciences. Stanford University. 1978. 
(12) Dolsak, B. y Jezernik, A.: "Mesh generation expert system for engineering analyses with FEM", Computers in Industry, vol. 17 (1991), p. 6.

(13) Turkiyyah, G. M. y Fenves, J. S.: "Knowledge-Based Assistance for Finite-Element Modeling", IEEE Expert, (1996), pp. 23-32.

(14) Hartmann, D. y Lehner, K.: "Non-numerical modeling techniques in structural optimization", Structural and Multidisciplinary Optimization, vol. 4 (1992). Doi: 10.1007/ BF01742740.

(15) Mukherjee, A. y Deshpande, J. M.: "Application of artificial neural networks in structural design expert systems", Computers and Structures, vol. 54 (1995), p. 8.

(16) Yoshimura, S.: "Automated structural design based on knowledge engineering and fuzzy control", Engineering Computations, vol. 12 (1995), p. 15.

(17) Bozzo Rotondo, L. M.: Qualitative Reasoning for earthquake resistant buildings, Centro Internacional de Métodos Numéricos en Ingeniería, España, 1993, p. 149.

(18) Álvarez Romero, S. O.; Baeza Pereyre, J. R.; Ceron Gil, M.: An Expert System for Scheduling Execution of Massive Housing Projects, 1998, citado 2010; disponible en: http:// www.ingenieria.uady.mx/weblioteca/sistemasinteligentes/Tema01/CIBW107AlvarezBaezaCeronV2.htm.

(19) Pérez Echazábal, L.: Influencia del medio ambiente en la patología de los monumentos de alto valor histórico construidos con materiales pétreos naturales, Instituto Superior Politécnico "José Antonio Echevarría", La Habana, 2000.

(20) Esbert, R. M. et al.: Manual de diagnosis y tratamiento de materiales pétreos y cerámicos, Colegio de aparejadores y arquitectos técnicos de Barcelona, Barcelona, 1997.

(21) Álvarez Rodríguez, O.: Patología, Diagnóstico y Rehabilitación de Edificaciones, monografía, La Paz (2003).

(22) Álvarez Rodríguez, O.: Muros de fábricas. Tipologías constructivas. Principales deterioros. Técnicas de intervención, Instituto Superior Politécnico "José Antonio Echevarría", La Habana, 2003.

(23) Autores, C. D.: Curso de Rehabilitación 7, COAM, Madrid, 1988.

(24) Menéndez Menéndez, J.: Desperfectos en construcciones de ingeniería y arquitectura (resumen de veinte conferencias), Centro de Información de la Construcción, La Habana, 1986.

(25) Ortega Andrade, F.: La Fábrica pétrea y sus patologías, Gran Canaria, España, 1999, cap. 3.

(26) Olivera Ranero, A.: Patologías de los materiales de la Construcción, Universidad Central "Marta Abreu" de Las Villas, Santa Clara, 2005, p. 11.

(27) Monjo Carrió, J.: Tratado de Rehabilitación, Universidad Politécnica de Madrid, Madrid, 1998, vol. 3.

(28) Eldridge, H.: Construcción. Defectos comunes, G. Gili, Barcelona, 1982.

(29) Menéndez Menéndez, J.: Desperfectos en construcciones de ingeniería y arquitectura: Diagnóstico, reparaciones, reconstrucciones, Científico-Técnica, La Habana, Cuba, 1986.

(30) Ruiz, G. y Hernández, E.: Apuntes de rehabilitación de edificios, ONG Sur, Madrid, 1995, vols. 1, 2, 3.

(31) Autores, C. D.: Tratado de Rehabilitación, Murilla-Lería, Madrid, 1999, vol. 3.

(32) Autores, C. D.: Tratado de Rehabilitación, Murilla-Lería, Madrid, 1999, vol. 4.

(33) Ortega Andrade, F.: Humedades en la edificación, Editan, S.A., Sevilla, 1989.

(34) Coscollano Rodríguez, J.: Tratamiento de las Humedades en los Edificios, International Thompson, Madrid, 2000.

(35) Más-Guindal Lafarga, A. J.: "Las grietas en las estructuras de fábrica. Un procedimiento para evaluar la forma de trabajo de estas", Informes de la Construcción, vol. 48, n 446 (1996). Doi: 10.3989/ic.1996.v48.i446

(36) Bautista Carrascosa, S.; Martín de la Morena, C. y Mieres Royo, J. M.: "Recomendaciones técnicas para la reducción de patologías en el terrazo", Informes de la Construcción, vol. 49, nº 454 (1998).

(37) Rincón, J.M.; Romero, M.: "Fundamentos y clasificación de las esflorescencias en ladriIlos de construcción", Materiales de Construcción, n 260 (2000).

(38) Doménico, L.: Iniciación a las restauraciones pétreas, Caja General de Ahorros de Granada, Granada, 1991.

(39) Bonshor, R. B.: Cracking in buildings, Construction Research Communications Ltd, 1996.

(40) Broto, C.: Enciclopedia Broto de Patologías de la Construcción, Links Internacional, 2009, p. 1389.

(41) Pentón Piña, E.: Patologías y Tratamiento de los materiales pétreos naturales utilizados en la construcción de edificaciones, Universidad Central "Marta Abreu" de Las Villas, Santa Clara, 2007.

(42) Figueredo Mariño, L.: Fichas técnicas. Diagnosis y tratamientos de las patologías inherentes a los materiales cerámicos en la construcción de edificaciones, Universidad Central "Marta Abreu" de Las Villas, Santa Clara, 2008. 